animal), streptococcal cell wall in IA $(5 \mu \mathrm{g} / 50 \mu \mathrm{l} / \mathrm{animal})$. The animals were injected on day 10 and monitored for 21 or 28 days. Visual evaluation of the severity of arthritis and histological lesions were performed.

Results: Coll2-1 at $0.45 \mathrm{nmol}\left({ }^{* *} \mathrm{p}<0.01\right)$ and $4.5 \mathrm{nmol}\left({ }^{*} \mathrm{p}<0.05\right)$ significantly increased IL-8 gene expression and tended to increase VEGF expression by synoviocytes. With AS0619, a specific antiserum for Coll2-1 peptide, IL-8 expression significantly decreased $\left({ }^{*} p<0.05\right)$. Coll2 -1 also induced both translocation of $p 65$ and IkB- $\alpha$ degradation. The latter being reduced with oxidative stress inhibitors. With CLI-095, we observed a decrease of IL-8 expression. In vivo, bovine type II collagen injection and Coll2-1 peptide injection resulted in an increase in visual arthritis score from D7. The global histological score was also increased by bovine type II collagen on D21 $(p=0.0005)$ and on D28 $(p<0.0001)$ and by the peptide Coll2-1 on D21 at the concentration of $200 \mu \mathrm{g}(\mathrm{p}=0.0252)$ and on D28 at the concentration of $20 \mu \mathrm{g}(\mathrm{p}=0.0025)$. Compared to control, all the components of the histological score were similarly modified by bovine type II collagen and the peptide Coll2-1 at both on D21 and on D28: increase in the inflammatory parameter (D21 $200 \mu \mathrm{g} \mathrm{p}=0.0217$ and D28 $20 \mu \mathrm{g} \mathrm{p}=0.0021$ ), reduction of proteoglycan contents (D28 $200 \mu \mathrm{g} \mathrm{p}=0.0072$ and $20 \mu \mathrm{g} \mathrm{p}=0.0024$ ), increased cartilage degradation (J28 $200 \mu \mathrm{g} \mathrm{p}=0.0070$ and $20 \mu \mathrm{g} \mathrm{p}=0.0024$ ) and modification of the subchondral bone (D21 $200 \mu \mathrm{g} \mathrm{p}=0.0025$ and D28 $20 \mu \mathrm{g} p=0.0065$ ). Similarly, both the injection of SCW and that of Coll2-1 peptide induced an increase in the visual score from D10. The effect of Coll2-1 peptide on this score was identical to that of the SCW. Compared to control, SCW and Coll2-1 peptide increased the global histological score (D21 p=0.0119 and D28 p=0.0045). Like SCW, the injection of Coll2-1 peptide caused both inflammatory reaction, loss of proteoglycan, appearance of cartilage structural lesions (D28 $0.5 \mu \mathrm{g} \mathrm{p}=0.0201$ ) and subchondral bone modification.

Conclusions: Coll2-1 peptide is able to induce an inflammatory reaction and structural changes in articular cartilage and subchondral bone comparable to those induced by SCW and bovine type II collagen. In conclusion, Coll2-1 may initiate nonspecific natural immunity and therefore be a therapeutic target for biotherapy.

Disclosure of Interest: None declared

DOI: 10.1136/annrheumdis-2018-eular.1036

\section{SAT0053 IDENTIFICATION OF NOVEL DRUGS WITH SENOLYTIC ACTIVITY AS OSTEOARTHRITIS THERAPEUTICS}

U. Nogueira-Recalde ${ }^{1}$, F.J. Blanco ${ }^{1}$, M.I. Loza ${ }^{2}$, D. Grassi ${ }^{3}$, P. Robbins ${ }^{3}$, E. Dominguez ${ }^{2}$, B. Carames ${ }^{1} .{ }^{1}$ Rheumatology Division. Cartilage Biology Group, The Institute of Biomedical Research of A Coruña (INIBIC), A coruña; ${ }^{2}$ Biofarma Research Group, Center for Research in Molecular Medicine and Chronic Diseases (CIMUS), University of Santiago de Compostela, Santiago, Spain; ${ }^{3}$ Department of Metabolism and Aging, The Scripps Research Institute, Jupiter, FL, USA

Background: Disease-modifying treatments for Osteoarthritis (OA) are not available. Aging-related features such as failure of cellular homeostasis mechanisms, including autophagy, cause extracellular matrix damage, chondrocyte senescence and death, which leads to articular cartilage degeneration as well as loss of joint function.

Objectives: The objective of this study was to identify senolytics and activators of autophagy by cell-based imaging of approved drugs in human chondrocytes.

Methods: To induce cellular senescence and reduced autophagy, High Content Screening system. Validation assays with readouts for senescence, autophagic flux, inflammation and apoptosis in primary human chondrocytes were performed. The anabolic effect on human cartilage and chondrocytes was evaluated by Safranin O staining and Nitric oxide production. To define the effects on senescence (senomorphic or senolytic), TC28a2 chondrocytes and human lung fibroblasts (IMR90) were employed. Senescence was induced in TC28a2 and IMR90 by treatment with IL-6 $(20 \mathrm{ng} / \mathrm{ml})$ for 72 hours and Etoposide $(20 \mu \mathrm{M})$ for 48 hours, respectively, and treated with serial dilutions of identified compounds. The number of senescence cells and the number of total cells were determined with Cell Analyzer 6000 Confocal Imaging System. Navitoclax $(2,5 \mu \mathrm{M})$ and Rapamycin $(10 \mu \mathrm{M})$ were employed as reference controls for senolytic and senomorphic effects, respectively.

Results: Our primary screen yielded 279 senotherapeutic compounds. The effects of hits at inducing the autophagic flux were evaluated. 37 compounds with both senotherapeutic and pro-autophagy effects were selected. An approved drug with a defined molecular mechanism of action was selected for further validation. The compound reduced senescence $(p<0.001)$ and increased autophagic flux $(p<0.0001)$. Furthermore, we found that it protects against defective autophagy and inflammation in response to IL-6 and IL-1b. This protective effect was confirmed in human cartilage explants by a reduction of proteoglycans loss $(\mathrm{p}<0.05)$ and in primary human chondrocytes by a reduction of NO production and chondrocyte death by apoptosis $(p<0.0001)$. Moreover, a significant senolytic effect of the selected compound was observed in both chondrocytes and fibroblasts $(p<0.05)$. This effect was also observed for structurally different compounds sharing the same mechanism of action, suggesting that pharmacological modulation of this mechanism may provide therapeutic benefits in OA.

Conclusions: Our imaging screening methodology provides a unique opportunity to identify drugs and mechanisms to prevent cartilage pathology. Autophagy activation and disruption of senescence may provide benefits for delaying cartilage degeneration.

Acknowledgements:

Disclosure of Interest: None declared

DOI: 10.1136/annrheumdis-2018-eular.3680

\section{SAT0054 SIGNIFICANT DECREASE OF T-CELLS BUT NOT MACROPHAGES IN THE SYNOVIUM OF PATIENTS WITH ACTIVE RHEUMATOID ARTHRITIS AFTER TREATMENT WITH TOCILIZUMAB}

K. Chatzidionysiou, M. Engström, E. af Klint, A. Hensvold, A.I. Catrina. Department of Rheumatology, Karolinska University Hospital, Karolinska Institute, Stockholm, Sweden

Background: Tocilizumab (TCZ) is an anti-IL6R monoclonal antibody approved for the treatment of Rheumatoid Arthritis (RA). There is limited data on synovial tis sue histology changes.

Objectives: The aim of this study was to evaluate the effect of TCZ on synovial cell populations and on citrullination.

Methods: 15 patients with RA, according to ${ }^{A C R} 1987$ criteria, independent of disease duration, were included. Synovial biopsies were obtained before and after 8 weeks of treatment. We evaluated by immunohistochemistry $(\mathrm{IHC})$ expression of citrullinated proteins (CP) and protein arginine deiminase (PAD) enzymes in synovial tissue before and after treatment (1325:C03, 1325:B09, PAD2, PAD4). Negative controls were used for each antibody. Expression of CD68, CD3, CD20 and CD55 was also evaluated. Evaluation of all IHC variables was performed by two blinded independent observers using a semiquantitative score on a $0-3$ scale $(0$, no staining; 1 , low amounts of staining; 2 , moderate amounts of staining; 3 , high amounts of staining). Paired-wised Wilcoxon Signed Ranks Test was used to compare the median values.

Results: The median (IQR) age, disease duration, N. prior biologic DMARDs and DAS28 at baseline was $66,{ }^{58-79} 4(1-13), 1(0-2), 6(5-7)$, respectively. $93 \%$ were female, $53 \%$ were RF +and $60 \%$ ACPA+, $53 \%$ had concomitant glucocorticoids and only $27 \%$ had concomitant conventional synthetic DMARDs. Significant reductions in DAS28, swollen and tender joint count (SJC and TJC, respectively) and acute phase reactants (ESR and CRP) were observed between baseline and 8 weeks (table 1). By IHC, TCZ induced significant decrease in the number of CD3, C03 and CD55, but not in the number of the other CP, PAD2, PAD4 and CD68 (table 1).

Abstract Sat0054 - Table 1

Baseline 8 weeks p-value

(Wilcoxon Signed Ranks Test)

DAS28 BL 5.9 (4.7-6.8) 2.98 (2.0-3.8) 0.028

SJC BL 9 (3-14) $1(0-4.25) 0.009$

TJC BL $10^{4-15} 1(0-2.25) 0.017$

ESR BL 34 $34^{15-69} 6(5-16) 0.001$

CRP BL $11^{5-27} 1(1-2) 0.005$

CD68 2 (1-3) $2(0.75-3) 0.41$

CD3 2 (1-3) 1 (0-2) 0.046

CD55 2 (2-3) $1.5(1-2) 0.05$

C03 (cit) 1 (0-1) $0(0-1) 0.025$

B09 (cit) $2(1-2) 2(1-2) 0.20$

PAD2 $3(2-3) 2(1.5-3) 0.16$

PAD4 $2(2-3) 2(2-3) 0.37$

Conclusions: Treatment with tocilizumab reduced the number of synovial tissue T cells. This was observed in the responders but not in non-responders. Significant reduction in citrullination as assessed by expression of 1325:C03 but not 1325:B09. No significant reduction in macrophages was observed.

Acknowledgements: This study was funded by Roche.

Disclosure of Interest: None declared

DOI: 10.1136/annrheumdis-2018-eular.5459 